\title{
La enseñanza virtual de contabilidad, retos de universidades de la región centro del Perú
}

\author{
Wilder Sergio Hilario Aquino1, Marcos Martin Bustios Martínez² \\ ${ }^{1}$ Universidad Continental, Perú \\ ${ }^{2}$ Universidad Continental, Perú
}

\begin{abstract}
Resumen
La enseñanza de Contabilidad en el marco del confinamiento COVID-19 en el año 2020 tuvo un inicio forzado y con cierto retraso, con muchos retos y adecuaciones de manera general en infraestructura, a nivel docente, de tecnologías y trabajadores administrativos. Todos ellos obstáculos que las universidades privadas pudieron sortear con mayor facilidad y con los recursos que ameritaba la coyuntura. Estos cambios partieron a partir de la exigencia de la entidad supervisora del sistema universitario peruano, SUNEDU, la cual requería el inicio de clases y el cumplimiento del programa o calendario académico que correspondía a los años 2020-2021. Por lo tanto, las universidades y los programas debían adecuar sus ofertas y acciones ante el nuevo escenario de la educación y formación de futuros contadores. Para ello, se implementaron nuevas plataformas, aulas virtuales, estrategias y evaluación, y se capacitaron a los docentes y el personal administrativo con la finalidad de cumplir con los estándares requeridos por la norma y el mercado. El presente trabajo pretende enunciar acciones y elementos necesarios para ayudar a la reflexión del estado de cada una de las facultades de Contabilidad y las acciones imprescindibles a ejecutar para las nuevas exigencias en la formación de los contadores.
\end{abstract}

Palabras clave: enseñanza virtual, retos de las universidades, Sunedu, Contabilidad, formación de futuros contadores.

\section{E-learning in accounting, challenges for universities in the central region of Peru}

\begin{abstract}
The teaching of Accounting in the framework of the COVID-19 confinement in the year 2020 had a forced start and with a certain delay, with many challenges and adaptations in general in infrastructure, teaching, technology and administrative workers. All of these were obstacles that the private universities were able to overcome more easily
\end{abstract}


and with the resources that the situation merited. These changes were based on the demands of the supervisory body of the Peruvian university system, SUNEDU, which required the start of classes and the fulfilment of the academic programme or calendar corresponding to the years 2020-2021. Therefore, universities and programmes had to adapt their offerings and actions to the new scenario of education and training of future accountants. To this end, new platforms, virtual classrooms, strategies and evaluation were implemented, and teachers and administrative staff were trained in order to meet the standards required by the regulation and the market. This paper aims to set out actions and elements necessary to help reflect on the state of each of the faculties of accounting and the essential actions to be taken to meet the new requirements in the training of accountants.

Keywords: virtual teaching, university challenges, Sunedu, Accounting, training of future accountants

\title{
O ensino virtual da contabilidade, desafios das universidades da região central do Peru
}

\begin{abstract}
Resumo
O ensino da Contabilidade no âmbito do confinamento COVID-19 no ano 2020 teve um início forçado e com algum atraso, com muitos desafios e ajustamentos de uma forma geral nas infra-estruturas, ao nível do ensino, das tecnologias e dos trabalhadores administrativos. Todos eles foram obstáculos que as universidades privadas conseguiram ultrapassar mais facilmente e com os recursos que a situação merecia. Estas mudanças partiram da exigência da entidade supervisora do sistema universitário peruano, SUNEDU, que exigiu o início das aulas e o cumprimento do programa ou calendário académico que correspondia aos anos 2020-2021. Por conseguinte, as universidades e programas tiveram de adaptar as suas ofertas e acções ao novo cenário de educação e formação de futuros contabilistas. Para o efeito, foram implementadas novas plataformas, salas de aula virtuais, estratégias e avaliação, e foram formados professores e pessoal administrativo a fim de satisfazer os padrões exigidos pela norma e pelo mercado. Este documento visa enunciar acções e elementos necessários para ajudar a reflectir o estado de cada uma das faculdades de Contabilidade e as acções essenciais a implementar para as novas exigências na formação dos contabilistas.
\end{abstract}

Palavras-chave: ensino virtual, desafios universitários, Sunedu, Contabilidade, formação de futuros contadores.

\section{Introducción escenario regional}

El año 2020 con el inicio del confinamiento el inicio de las clases de las facultades de Contabilidad se vio interrumpida o postergada, se presenta un inventario de universidades, facultades, escuelas o programas de Contabilidad (TUNI, 2021): 


\section{Tabla 1. Cantidad de alumnos en la carrera de Contabilidad en universidades licenciadas, Departamento de Junín.}

\begin{tabular}{|l|l|l|l|}
\hline Universidad & \multicolumn{1}{|c|}{ Nombre } & Modalidad & \\
\hline $\begin{array}{l}\text { Universidad } \\
\text { Continental }\end{array}$ & $\begin{array}{l}\text { EAP } \\
\text { Contabilidad }\end{array}$ & Presencial & 1572 \\
\hline $\begin{array}{l}\text { Universidad } \\
\text { Nacional del } \\
\text { Centro del Perú }\end{array}$ & $\begin{array}{l}\text { Facultad de } \\
\text { Contabilidad }\end{array}$ & Presencial & \multirow{2}{*}{952} \\
\hline $\begin{array}{l}\text { Universidad } \\
\text { Peruana los Andes } \\
(*)\end{array}$ & $\begin{array}{l}\text { EAP } \\
\text { Contabilidad y }\end{array}$ & Pranzas & \\
\hline
\end{tabular}

Fuente: TUNI (2021)

Nota: $\left(^{*}\right)$ No cuenta con información. Se indagó con la finalidad de obtener la población total.

Se evidencia de una la población universitaria en pregrado Contabilidad 3,435 en la Región Junín, al 2016 de universidades licenciadas, se puede afirmar que la tendencia al 2021, con una población cercana a 4,000 estudiante de Contabilidad de las modalidades presencial y semipresencial, puesto que al 2019 se autoriza a la filial de la Universidad Tecnológica del Perú en la ciudad de Huancayo.

\section{Normatividad vigente}

Desde el ámbito normativo, en el escenario de la pandemia, SUNEDU emite el Decreto Legislativo № 1496 de fecha 09 de mayo de 2020, el cual establece disposiciones en materia de educación superior universitaria en el marco del estado de emergencia sanitaria a nivel nacional. En ella se norma las nuevas modalidades de estudios universitarios: presencial $\downarrow$ hasta el $20 \%$ de los créditos del programa académico $\downarrow$, semi-presencial $\downarrow$ hasta el $70 \%$ de los créditos del programa académico $\downarrow y$ a distancia o no presencial $\downarrow$ puede superar el $80 \%$ en los programas para una población mayor de 24 años $\downarrow$. Con ello se modifica el artículo 47 de la ley $N^{\circ} 30220$, que dispone además el uso de entornos virtuales en cualquiera de las tres modalidades referidas, y esta deberá ser compatible con los contenidos, competencias esperadas, metodología de enseñanza, forma de evaluación y la modalidad de los cursos de cada programa académico (Decreto Legislativo N.o 1496, 2020).

De cara a esta circunstancia, en las universidades de la región Junín existía una necesidad de plataformas relacionadas a repositorios, video clases, capacitación a los docentes en la gestión de tecnología de información y comunicación - TIC, 
recursos tecnológicos y conectividad de un número considerable de alumnos. Estas limitaciones o necesidades de la comunidad universitaria fueron requeridas a raíz del desarrollo de las clases virtuales, cosa que las universidades tuvieron que resolver en el corto plazo y que condujo a que se iniciaran las clases recién en los meses de abril y mayo.

\section{Tendencia de la enseñanza virtual en Latinoamérica}

Autores como Fainholc realizan una mirada sobre la necesidad de la enseñanza virtual universitaria:

El sistema educación virtual, en especial de nivel superior direccionado para la producción de conocimiento en América Latina, si bien ha demostrado avances, aún presenta graves debilidades con una baja incorporación de Internet, TIC y derivados. No superó la baja calidad educativa general y a distancia, no interviniendo positivamente con sus impactos en la vulnerabilidad y riesgos sociales existentes $(2016$, p. 1).

Fainholc (2016) en su estudio señala la secuencia de las épocas y afirma que la etapa "híper-moderna" se caracteriza por estar acompañada de grandes crisis sociales, económicas y sobre toda las educativas. Sin embargo, en estas etapas es donde las TICs juegan un rol importante, ya que permitirían, en un contexto como el latinoamericano, una comunicación más eficiente por medios electrónicos. No obstante, estas ventajas chocan con la realidad de nuestra región, la cual tiene un incipiente desarrollo. En ese sentido, los espacios educativos universitarios de la región tuvieron que adaptarse rápidamente a la virtualidad: algunos se adaptaron rápidamente, otros con mayor lentitud $\downarrow$ a partir de herramientas como Moodle, MOOC, entre otros $\downarrow$, pero otros no, ya sea por la carencia de recursos o la falta de aceptación de la educación virtual.

Para el desarrollo de la educación en plataformas virtuales se recomendó una modalidad mixta, lo cual demanda una revisión de las formas tradicionales de enseñanza, una formación profesionalizada, actualización y renovación permanente de los conocimientos e y métodos de investigación. En otras palabras, este cambio requiere que los actores cuenten con las condiciones adecuadas para cumplir con los roles que les toca desempeñar.

Uno de los elementos a desarrollar por parte de los docentes son las habilidades en las TIC. Estas ayudarían a flexibilizar la educación superior tomando en cuenta el nuevo contexto de pandemia, y permitiría una adecuación de la estructuración de los contenidos, en tiempos, espacios de comunicación y en la evaluación sincrónica y asincrónica. Asimismo, ayudaría en la enseñanza de una lectura crítica y una escritura estratégica en cualquier formato: computadora, celular, tableta, entre otros. Para ello será necesario un diagnóstico institucional, para preservar y desplegar la participación de todos los estamentos de un programa educativo universitario, y 
todo ello a través de programas de aprendizaje electrónico puro $\downarrow$ e-learning $\downarrow$ o mixtos $\downarrow$ blended learning $\downarrow$.

\section{Plataformas en la enseñanza en entornos virtuales}

Sobre el uso de las plataformas digitales en los sistemas universitarios, autores como De Pablos, Colás, López y García-Lázaro (2019) mencionan que en España y Europa las plataformas digitales nacieron en los años noventa, y tienen la función de actuar como elementos facilitadores del proceso de enseñanza-aprendizaje en el ámbito educativo, preferentemente en el universitario.

Estas plataformas digitales y los procesos de enseñanza-aprendizaje en las universidades han incorporado en las últimas dos décadas cambios sustantivos, tanto en el terreno tecnológico como pedagógico y modelos educativos más actualizados y acordes con teorías educativas más fundamentadas. A ello se califica como"ecosistemas tecnológicos universitarios" como una nueva cultura pedagógica universitaria. Además, se afirma que las plataformas digitales evidencian los ecosistemas tecnológicos y ecologías del aprendizaje, ello con una prospectiva de la transformación tecnológica de las plataformas orientada a un aprendizaje escalable y sostenible; algunas de las más usadas son Blackboard, Google Meet, Zoom, Microsoft Teams, entre otros (De Pablos et al., 2019).

En el contexto de la pandemia, la necesidad de las universidades de la región y del país por cumplir con el calendario académico impulsó un viraje y fortalecimiento de la enseñanza virtual. Chingel (2021), en el libro 15 habilidades y herramientas web para ser un docente innovador, detalla estos aspectos:

a. Plataforma: entorno de hardware y software que permite gestionar el desarrollo de actividades formativas de un curso virtual.

b. Contenidos: es la información que forma parte del curso virtual. Para la elaboración de materiales se deben tener en cuenta lo siguiente: adecuación de contenidos según las necesidades y posibilidades del estudiante; mejor calidad que cantidad de información presentada. Estructura y organización de contenidos adecuado.

c. Herramientas comunicativas: estas herramientas permiten la interacción entre los agentes del proceso enseñanza-aprendizaje, a través de foros, chat, videoconferencias, entre otros.

\section{Estrategias en la enseñanza en entorno virtuales}

En cuanto a estrategias de trabajo colaborativo y de aprendizaje en entornos virtuales, jóvenes universitarios todavía deben desarrollar competencias para el aprendizaje autónomo; esto se debe a que los estudiantes vienen de una enseñanza tradicional con el rol protagónico del docente en el aula. 
En tanto, Espinoza y Rodríguez (2017) muestran los resultados de su investigación, en la que demuestran que el papel del docente en los ambientes virtuales es muy importante, ya que genera una nueva cultura de aprendizaje y promueven una relación pedagógica equilibrada que permite la habilidad y la confianza para el aprendizaje autónomo y colaborativo.

Asimismo, Rene y Leonor (2017) señalan la necesidad de un diálogo y un trabajo colaborativo entre alumnos y docentes, ya que si bien varios estudiantes son nativos digitales $\downarrow$ pues tienen la habilidad de búsqueda de información y el uso de equipos, plataformas y medios de comunicación $\downarrow$, requieren del aprendizaje y el conocimiento de herramientas de búsqueda, análisis y discernimiento.

\section{Evaluación en el entorno de enseñanza virtual}

La enseñanza virtual y las evaluaciones que ella trae deben ser vistas como un recurso que permite el desarrollo de habilidades y destrezas, así como una herramienta de apoyo en la labor educativa que permite seguir, controlary regular los conocimientos (Gómez \& Jiménez, 2011).

En ese sentido las TICs aportan entornos y herramientas que amplían considerablemente las posibilidades de trabajo en distintos ambientes grupales y sincrónicos-asincrónicos, con procedimientos, herramientas y formas de evaluar los conocimientos mediante procesos puramente descriptivos o conceptuales (exámenes, pruebas de destreza, problemas teóricos). Permite el surgimiento de procedimientos y métodos donde se simulan situaciones reales y casos prácticos, con metodologías de indagación, de investigación formativa, con elaboración de proyectos, entre otros. Asimismo, a partir de herramientas como la "evaluación en la red" o la "evaluación online", el profesor puede seguir el desenvolvimiento del alumno, la forma cómo ejecuta las tareas y resuelve los problemas.

Adicionalmente, la enseñanza virtual permitió el surgimiento de otros tipos de evaluación, como la automática, la de trabajos monográficos y proyectos, la evaluación colaborativa y la evaluación interactiva. Sin embargo, se corre el riesgo de la copia, plagio y otros fraudes. Para evitar ello, Zapata (2010) menciona estrategias como el apelar a los principios o valores, establecer reglas en el proceso de evaluación y la aplicación de sanciones propias de las asignaturas y los reglamentos.

\section{Conclusiones}

- La realidad de las universidades en la región de Junín era que ejecutaban los procesos de enseñanza y aprendizaje de manera presencial, indistintamente de las modalidades que la Ley $\mathrm{N}^{\circ} 30220$ autorizaba, porque no contaban con una plataforma de trabajos asíncronos o repositorios de información, oficial o formal. Esto demuestra que la realidad peruana está distante de la realidad latinoamericana y mundial, donde la educación superior tiende a la virtualidad 
de sus servicios. Por lo tanto, se espera que las autoridades universitarias apuesten por mejorar sustancialmente las condiciones tecnológicas, infraestructura, humanas -docentes y administrativos- en las universidades, por medio de convenios, intercambios virtuales, y adquisición y sostenibilidad de la proforma virtual -al margen del levantamiento del confinamiento sanitario-. Esto permitirá a docentes, estudiantes y colaboradores estar acorde a los cambios o mejoras en el mundo.

- La pandemia ha demostrado, en el caso de las universidades que pudieron implementarlo, un uso adecuado e integral de las tecnologías de información y comunicación aplicables a la educación superior universitaria-TIC. A la fecha, han transcurrido tres ciclos desde este proceso de readaptación, y las universidades demostraron una adecuada selección y ampliación del uso de las plataformas existentes de acuerdo a las necesidades e intereses institucionales. Por lo tanto, el reto implica que las demás instituciones que no han podido adaptarse aún a la virtualidad puedan planear el uso de las TICs para la enseñanza virtual o mixta, y para las nuevas exigencias del futuro, el cual estará más conectado al mundo.

- Las universidades y programas de Contabilidad, en lo referente a estrategias didácticas, deben de realizar el cambio de una clásica modalidad presencial y del tipo magistral, a una enseñanza experiencial y colaborativa. Ello se lograría a través de la combinación del uso intensivo de TICs. Para ello no solo se requiere contar con un laboratorio de cómputo y de una conexión remota, sino también brindar paquetes o programas informáticos que permitan a los egresados desarrollar competencias como usuarios, gestores y auditores en las áreas de la contabilidad financiera, tributación, costos, auditoria y finanzas. Esto permitirá un perfil de egreso que desarrolle habilidades en el uso de herramientas tecnológicas y el desarrollo de habilidades blandas para una correcta e inmediata incorporación al ámbito laboral dependiente e independiente.

- Un aspecto importante en estas clases virtuales fue la adaptación de los modelos de evaluación. Tanto docentes como estudiantes estaban acostumbrados a las clásicas mediciones de pruebas escritas con preguntas abiertas, selección múltiple y ejercicios, donde no se podían utilizar elementos de consulta o ayudas visuales. Pero ello debió ser revisado porque los nuevos sistemas de educación y evaluación son en línea, lo que facilitaría a los alumnos usar buscadores, fuentes y otros medios disponibles. Por eso, se ha optado por incentivar en las evaluaciones un análisis crítico, el uso de otras herramientas como los formularios, los ensayos, y la ejecución de cálculos. A ello se le debe complementar la aplicación de un reglamento académico estricto que penalice el plagio o la copia, con la finalidad de educar a los estudiantes en el respeto de la autoría. 


\section{Referencias bibliográficas}

Chingel, G. C. (2021). 15 Hablidades y Herramietas web para Ser Docente Innovador. Chiclayo: Instituto de Tecnologías para la Educación (ITED).

de Pablos, J., Colás, M., López Gracia, A., \& García Lázaro, I. (2019). Los usos de las plataformas digitales en la enseñanza universitaria. Perspectivas desde la investigación educativa . Revista de docencia universitaria, 14. Obtenido de https:// www.ride.org.mx/index.php/RIDE/article/view/274

Espinoza, L., \& Rodríguez, R. (2017). La generación de ambientes de aprendizaje: un análisis de la percepción juvenil. Revista lberoamericana para la Investigación y el Desarrollo educativo, 23.

Fainholc, B. (30 de 01 de 2016). Presente y futuro latinoamericano de la enseñanza y el aprendizaje en entornos virtuales referidos a educación universitaria. RED-Revista de educación a distancia, 1 - 22. Obtenido de

Peruano, D. e. (nueve de mayo de 2020). Decreto Legislativo N.o 1496. Lima, Lima, Perú.

Rene, R., \& Leonor, E. (22 de Marzo de 2017). RIDE-Revista Iberoamerica para la Investigación y Desarrollo Educativo. Obtenido de https://www.ride.org.mx/index. php/RIDE/article/view/274

TUNI (2021). Sistema de educación universitaria. Obtenido de https://www.tuni.pe/ programas?term $=$ contabilidad\&page $=1$

Zapata, M. (05 de Setiembre de 2010). La evaluación en el contexto del Espacio Europeo de Educación Superior. RED-Docencia universitaria en la Sociedad del Conocimiento, 1-18. Obtenido de http://www.um.es/ead/reddusc/1

Fecha de recepción: 05/04/2021

Fecha de aceptación: 31/05/2021

Correspondencia: whilario@continental.edu.pe, mbustios@continental.edu.pe 